\title{
Estudo Comparativo do Desempenho de Imagens por Ressonância Magnética, Mamografia e Ecografia na Avaliação de Lesões Mamárias Benignas e Malignas Comparative Study of the Performance of Magnetic Resonance Imaging, Mammography and Ultrasonography in the Evaluation of Benign and Malignant Mammary Lesions
}

\author{
Carolina Moreira Maulaz ${ }^{1}$, Bruna Bressan Valentini ${ }^{2}$, Ana Maria Marques da Silva ${ }^{1}$, \\ Ricardo Meurer Papaléo ${ }^{1}$ \\ ${ }^{1}$ Núcleo de Pesquisa em Imagens Médicas, PUCRS, Porto Alegre, Brasil \\ ${ }^{2}$ Hospital Moinhos de Vento (HMV), Porto Alegre, Brasil
}

\begin{abstract}
Resumo
O câncer de mama é o tipo de câncer mais comum entre as mulheres no Brasil e no mundo. A mamografia é o exame considerado padrão ouro para rastreamento de câncer de mama e, tanto a ressonância magnética quanto a ultrassonografia, são utilizadas como complemento. Atualmente, existe um crescimento na quantidade de requerimentos médicos da ressonância como método de rastreamento de lesão, acompanhamento de tumores malignos e em casos de pacientes que possuam histórico familiar de câncer de mama. Este trabalho teve como objetivo comparar o desempenho das imagens por ressonância magnética com a mamografia e ecografia de mamas no diagnóstico de lesões mamárias malignas e benignas, utilizando a biópsia como padrão ouro. Foi feito um comparativo entre variáveis que quantificam características de desempenho, tais como sensibilidade, especificidade, valor preditivo positivo e negativo. Essas informações foram obtidas dos laudos médicos da biopsia e dos três métodos de aquisição de imagem. Os parâmetros de sensibilidade e especificidade para as lesões benignas foram de $91 \%$ e $63 \%$ para mamografia/ecografia e $77 \%$ e 100\% para ressonância magnética. Em relação às lesões malignas, a sensibilidade e especificidade encontradas foram de $57 \%$ e $100 \%$ para a mamografia e ecografia, respectivamente. Concluiu-se que a ressonância magnética é um bom exame para avaliação de lesões benignas, devido aos altos parâmetros de desempenho. Para as lesões malignas não foi possível obter os parâmetros, visto que os valores não foram válidos para o estudo.
\end{abstract}

Palavras-chave: ressonância magnética de mamas; mamografia; ecografia.

\begin{abstract}
Breast cancer is the most common type of cancer among women in Brazil and in the world. Mammography is the gold standard exam for breast cancer screening, and both magnetic resonance imaging and ultrasound are used as a complement. Currently there is a growing number of medical requirements for resonance as a method of tracking lesions, monitoring malignant tumors and in cases of patients with a family history of breast cancer. This study aimed to compare the performance of MRI with mammography and breast ultrasound in the diagnosis of malignant and benign breast lesions, using biopsy as the gold standard. A comparison was made between variables that quantify performance characteristics, such as sensitivity, specificity, positive and negative predictive value. The information was obtained from the medical biopsy reports and the three image acquisition methods. The sensitivity and specificity parameters for benign lesions were $91 \%$ and $63 \%$ for mammography/echography and $77 \%$ and $100 \%$ for magnetic resonance imaging. Regarding malignant lesions, the sensitivity and specificity found was $57 \%$ and $100 \%$ for mammography and ultrasound, respectively.. It was concluded that magnetic resonance imaging is a good test to evaluate benign lesions, due to high performance parameters. For the malignant lesions it was not possible to obtain the parameters, since the values were not valid for the study.
\end{abstract}

Keywords: magnetic resonance imaging of breasts; mammography; ultrasound.

\section{Introdução}

O câncer de mama é o tipo de câncer mais comum entre as mulheres no Brasil e no mundo. Ele é considerado a segunda maior causa de morte por doenças entre todas as mulheres e o primeiro entre os tipos de câncer. Para o ano de 2012, no Brasil, detectou-se cerca de 50 mil casos de câncer de mama entre as mulheres. Já no ano de 2016, a estimativa subiu para $57 \mathrm{mil}^{1}$. Essa estatística demonstra a gravidade deste tipo de câncer e a importância de detectá-lo precocemente. Por se tratar de uma lesão maligna, o melhor meio de detectar é através da imagem radiológica, podendo ser por ressonância magnética, ultrassonografia ou mamografia. 
A mamografia é o exame considerado padrão ouro $^{2}$ para rastreamento das lesões de mama, sendo realizado por um equipamento de raios $X$, conhecido como mamógrafo. O Ministério da Saúde recomenda, nas "Diretrizes para a Detecção Precoce do Câncer de Mama no Brasil" (2015), a realização de exame clínico das mamas para mulheres a partir de 40 anos, com frequência anual e, entre 50 e 69 anos, pelo menos uma mamografia de rastreamento a cada dois anos. Essa técnica de diagnóstico tem como principal objetivo a produção de imagens detalhadas de toda a estrutura interna da mama da paciente. As principais vantagens são a detecção precoce de determinadas alterações devido à alta resolução deste método, a alta disponibilidade em diversos hospitais e clínicas no Brasil e ao baixo preço atribuído ao exame. Por outro lado, as pacientes se queixam de desconforto durante o exame, ocorrendo casos em que a paciente sente uma dor tão intensa que não consegue realizar o exame e/ou o realiza de maneira inadequada, dificultando o diagnóstico.

A ecografia de mamas é um método de diagnóstico por imagem considerado o principal complementar à mamografia na detecção de alterações mamárias ${ }^{3}$. Sua característica fundamental reside na capacidade de distinguir cistos e lesões sólidas, algo difícil de detectar na mamografia. A biópsia, procedimento no qual se realiza coleta de células de tecido, é realizada por meio da ecografia. $O$ radiologista realiza a ecografia para visualizar a região da lesão e guiar durante o procedimento. Com esse material coletado, é possível identificar os aspectos anátomo-patológicos e, consequentemente, caracterizar a lesão em benigna ou maligna.

Um outro método de diagnóstico por imagem é a ressonância magnética $(\mathrm{RM})$ das mamas ${ }^{4}$. Ele foi utilizado pela primeira vez em 1986 e, desde então, passou por vários avanços. É um importante exame complementar para a mamografia, devido à sua alta sensibilidade e especificidade. Apesar da avaliação de implante mamário ser a maior indicação para realização da RM das mamas, o uso dessa técnica para avaliação de lesões está crescendo cada vez mais. Desde que se passou a utilizar o contraste de gadolínio $(\mathrm{Gd})$ nas suas aquisições, o desempenho da técnica aumentou significativamente, devido à rápida impregnação nas regiões com lesão maligna ${ }^{5}$.

A detecção precoce de lesões mamárias é prioridade. A realização rotineira de exames de diagnóstico por imagens é a melhor prevenção que as mulheres podem ter. Assim, obter um melhor conhecimento do desempenho de cada método para avaliação de lesão, contribuirá para um diagnóstico médico mais eficiente e preciso. Este trabalho teve como objetivo comparar o desempenho das imagens por ressonância magnética, mamografia e ultrassonografia no diagnóstico de lesões mamárias malignas e benignas, utilizando a biópsia como padrão ouro.

\section{Materiais e Métodos}

Neste trabalho, foi realizado um estudo retrospectivo de exames de mamografia e ecografia realizados no ano de 2016, em um hospital de grande porte de Porto Alegre, para os quais houve a solicitação de realização do exame de imagem por RM de mamas para fins de investigação de algum tipo de alteração. O projeto foi aprovado pelo comitê de ética com número CAAE 79532517.0.0000.5336.

Para realizar a comparação com a mamografia, ecografia e biópsia utilizaram-se apenas os casos em que a paciente havia realizado os exames com diferença de agendamento de seis meses, visto que não há alterações significativas na lesão neste intervalo de tempo. Com isso, o tamanho da amostra encontrado foi de 30 pacientes.

Os laudos investigados foram sempre de dois radiologistas: um para o laudo das imagens por RM e outro para o de mamografia e ecografia. Como em todos os casos as pacientes realizam a mamografia e ecografia no mesmo dia, os laudos foram feitos em conjunto, não podendo diferenciar as características analisadas em cada método individualmente. Portanto, as comparações a serem feitas serão da RM com a mamografia e ecografia.

As imagens foram adquiridas com o equipamento de mamografia utilizado do fabricante HOLOGIC, modelo Selênia. Os três ecógrafos utilizados são do fabricante Toshiba, modelo Xaria. Na RM foram utilizados equipamentos de 1,5T da marca Siemens, modelos Avanto (Avanto B17), Aera do ano de 2013 (Aera D13) e do Aera do ano de 2016 (Aera E11). Os parâmetros utilizados na aquisição de imagens por RM estão listados na Tabela 1.

Tabela 1. Parâmetros utilizados na aquisição de imagens por RM.

\begin{tabular}{|c|c|c|c|}
\hline & AVANTO B17 & AERA D13 & AERA E11 \\
\hline \multicolumn{4}{|c|}{ STIR } \\
\hline $\mathrm{TI}(\mathrm{ms})$ & 150 & 160 & 180 \\
\hline TR (ms) & 6000 & 6230 & 5790 \\
\hline $\mathrm{TE}(\mathrm{ms})$ & 77 & 73 & 74 \\
\hline Flip Angle & 150 & 150 & 170 \\
\hline Voxel $\left(\mathrm{mm}^{3}\right)$ & $1,1 \times 0,5 \times 3$ & $1 \times 1 \times 3$ & $0,9 \times 0,9 \times 4$ \\
\hline FoV $\left(\mathrm{mm}^{2}\right)$ & 280 & 330 & 330 \\
\hline Matriz $\left(\mathrm{mm}^{2}\right)$ & $256 \times 512$ & $288 \times 320$ & $269 \times 384$ \\
\hline \multicolumn{4}{|c|}{ T1 TSE } \\
\hline TR (ms) & 568 & 653 & 682 \\
\hline $\mathrm{TE}(\mathrm{ms})$ & 8,9 & 10 & 17 \\
\hline Flip Angle & 150 & 150 & 150 \\
\hline Voxel $\left(\mathrm{mm}^{3}\right)$ & $0,9 \times 0,5 \times 3$ & $0,6 \times 0,6 \times 3$ & $0,7 \times 0,7 \times 4$ \\
\hline $\mathrm{FoV}\left(\mathrm{mm}^{2}\right)$ & 280 & 330 & 330 \\
\hline Matriz $\left(\mathrm{mm}^{2}\right)$ & $307 \times 512$ & $307 \times 512$ & $269 \times 448$ \\
\hline \multicolumn{4}{|c|}{ DIFUSÃO } \\
\hline $\mathrm{TR}$ (ms) & 5600 & 6000 & 7700 \\
\hline $\mathrm{TE}(\mathrm{ms})$ & 91 & 74 & 92 \\
\hline Flip Angle & - & - & - \\
\hline
\end{tabular}




\begin{tabular}{llll} 
Voxel $\left(\mathrm{mm}^{3}\right)$ & $1,8 \times 1,8 \times 4$ & $2 \times 2 \times 4$ & $1,8 \times 1,8 \times 4$ \\
FoV $\left(\mathrm{mm}^{2}\right)$ & 340 & 340 & 340 \\
Matriz $\left(\mathrm{mm}^{2}\right)$ & $96 \times 192$ & $84 \times 170$ & $108 \times 192$ \\
& \multicolumn{3}{c}{ T1 DINÂMICO } \\
TR $(\mathrm{ms})$ & 3,67 & 3,58 & 5,14 \\
TE $(\mathrm{ms})$ & 1,41 & 1,49 & 2,39 \\
Flip Angle & 10 & 10 & 10 \\
Voxel $\left(\mathrm{mm}^{3}\right)$ & $1 \times 0,8 \times 1,3$ & $0,7 \times 0,7 \times 1,3$ & $0,9 \times 0,9 \times 1$ \\
FoV $\left(\mathrm{mm}{ }^{2}\right)$ & 340 & 330 & 360 \\
Matriz $\left(\mathrm{mm}^{2}\right)$ & $336 \times 448$ & $314 \times 448$ & $330 \times 384$ \\
& \multicolumn{1}{c}{ T1 ALTA RESOLUÇÃO } & \\
TR $(\mathrm{ms})$ & 11,9 & 4,86 & 8,84 \\
TE $(\mathrm{ms})$ & 5,95 & 2,46 & 4,6 \\
Flip Angle & 12 & 10 & 10 \\
Voxel $\left(\mathrm{mm}^{3}\right)$ & $0,6 \times 0,6 \times 0,9$ & $0,7 \times 0,7 \times 1$ & $0,7 \times 0,7 \times 0,9$ \\
FoV $\left(\mathrm{mm}^{2}\right)$ & 160 & 200 & 200 \\
Matriz $\left(\mathrm{mm}^{2}\right)$ & $256 \times 256$ & $202 \times 288$ & $202 \times 288$ \\
\hline
\end{tabular}

Os parâmetros de aquisição utilizados em mamografia estão listados na Tabela 2. Foi feita uma média dos valores de $\mathrm{kV}$, mAs, dose glandular por incidência (DG) e a compressão utilizada. Dentre as combinações ânodo/filtro disponíveis, tungstênio com ródio (W/Rh) foi a mais usada.

Tabela 2. Parâmetros de aquisição utilizados nos exames de mamografia por intervalo de variação.

\begin{tabular}{lcl}
\multicolumn{3}{c}{ mamografia por intervalo de variação. } \\
\hline Parâmetros & Valor médio & $\begin{array}{l}\text { Intervalo de } \\
\text { variação }\end{array}$ \\
\hline Ânodo/Filtro & W/Rh & W/Rh ou W/Ag \\
kV & 28 & $26-32$ \\
mAs & 139 & $41-309$ \\
DG (mGy) & 1,54 & $0,45-3,8$ \\
Compressão $(\mathrm{mm})$ & 58 & $32-114$ \\
\hline
\end{tabular}

Em relação aos parâmetros utilizados na ecografia, foram utilizados presets para a mamografia e um transdutor de $8 \mathrm{MHz}$. Nessa frequência, a resolução espacial axial $3 \lambda / 2$ é da ordem de $0,2 \mathrm{~mm}$ e a lateral $3 \lambda$ é de aproximadamente $0,4 \mathrm{~mm}$. Neste estudo, na amostra das 30 pacientes que realizaram as três técnicas, a menor lesão encontrada foi de $5 \mathrm{~mm}$.

Foram analisadas as alterações encontradas na mamografia, ecografia e ressonância magnética com os resultados anátomo-patológicos (biópsia). Nos laudos estão descritos detalhadamente os achados encontrados, tais como forma, formato de borda, diâmetro, localização e composição (nódulo sólido ou com conteúdo líquido), junto com a classificação BI-RADS. No laudo da biopsia é especificado o diâmetro da lesão (sendo utilizado sempre tamanho representado na ecografia, visto que esta é a técnica utilizada para guiar o procedimento), sua localização e o resultado histológico do anátomo-patológico.
A partir das características descritas e em conjunto com a classificação BIRADS, foi possível compará-las e determinar se a lesão possuía caráter benigno ou maligno em cada um dos quatro exames. Classificações BI-RADS da mamografia para lesões benignas variam do 1 até $4 \mathrm{~A}$ e lesões malignas variam do 4B até o 6 . Se for para a ressonância, lesões benignas possuem BI-RADS 1 até 3 e malignas de 4 a 6; nesse caso classificação BI-RADS 4 pode representar até $95 \%$ de suspeita de lesão maligna. Para mamografia, foi adotado este intervalo, uma vez que BI-RADS $4 \mathrm{~A}$ representa apenas 2 a $10 \%$ malignidade, sendo muito utilizada em suspeitas benignas. Lesões de caráter maligno possuem características bem evidentes nas imagens, sendo as principais palavras-chave o "formato espiculado" e "borda de forma irregular". Já os atributos que se destacam para alterações benignas são seu formato circunscrito e seu caráter cístico. Como no texto do laudo não está explícito o caráter da lesão, essas características associada ao tipo de lesão, em conjunto com a classificação BIRADS, foram essenciais para a identificar se a lesão era benigna ou maligna.

Identificando o tipo de lesão (maligna ou benigna), verificou-se se a alteração descrita em cada método de imagem estava conforme com o resultado da biópsia. Com essas informações, foi atribuído para cada exame se o resultado do laudo representava um verdadeiro positivo (VP), verdadeiro negativo (VN), falso positivo (FP) ou falso negativo (FN) para lesões maligna e/benigna. Assim, foi possível calcular a sensibilidade, a especificidade, valor preditivo positivo e valor preditivo negativo para cada método de imagem. Estas variáveis foram calculadas através das seguintes equações ${ }^{6}$ :

$$
\begin{aligned}
& \text { Sensibilidade }=\frac{V P}{V P+F N} \\
& \text { Especificidade }=\frac{V N}{V N+F P} \\
& \text { Valor preditivo positivo }=\frac{V P}{V P+F P} \\
& \text { Valor preditivo negativo }=\frac{V N}{V N+F N}
\end{aligned}
$$

Juntando-se as informações quantitativa e qualitativa das imagens por RM, da mamografia e ecografia, conseguiu-se realizar uma comparação do desempenho entre esses métodos de imagem.

\section{Resultados}

As estruturas anatômicas possuem a mesma composição independente da técnica, porém, cada tecido pode ser demonstrado de maneira diferente dependendo do método escolhido. Lesões benignas, por sua ampla variedade, podem ser mais difíceis de serem detectadas em relação as lesões malignas. 
Numa análise qualitativa das imagens de mamografia (Figura 1a), o carcinoma mamário apresenta uma região mais densa devido ao crescimento anormal do tecido, resultando numa maior atenuação da radiação no tecido. Nas imagens por RM de mamas, numa sequência T1 dinâmica (Figura 1b), o câncer também aparece hiperintenso por causa da sua alta vascularização e, consequentemente, com impregnação de contraste no tumor. Nota-se em ambos o formato alongado, não circunscrito, da lesão e suas bordas irregulares. Já na ecografia, devido à forte presença de líquido, o câncer de mama fica completamente hipoecóico na imagem (Figura 1c). Pode-se visualizar o tamanho encontrado da lesão na ecografia, imagem (c) da figura abaixo, utilizando o recurso da régua, sendo de $42,8 \mathrm{~mm}$ a distância de uma ponta a outra.

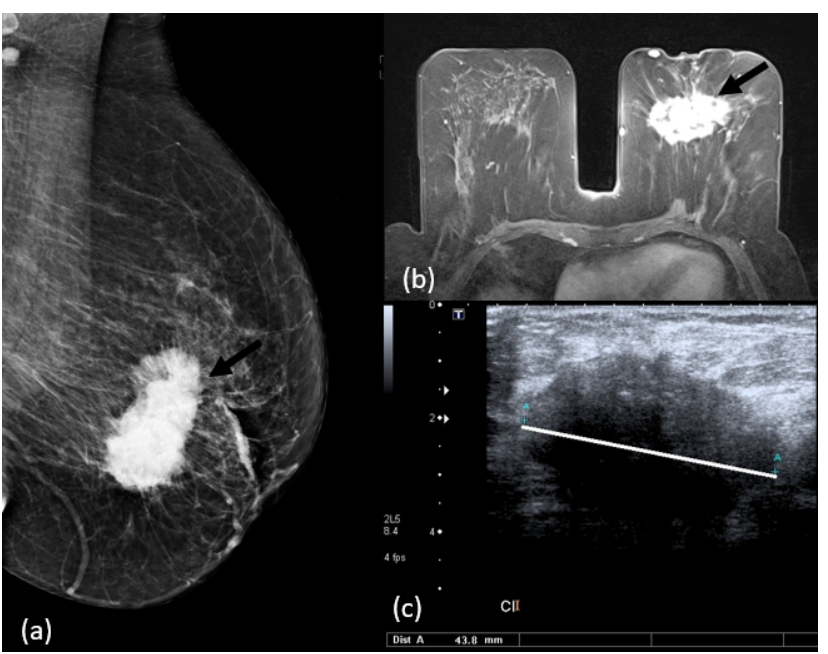

Figura 1. Imagens representativas de um câncer de mama sendo visto em diferentes métodos de aquisição de imagem. Nas imagens (a) e (b), mamografia e RM, respectivamente, a seta representa o carcinoma. Já na imagem (c), referente à ecografia, a reta branca indica o diâmetro da lesão. (Fonte: AUTOR, 2017)

As variedades das alterações benignas das mamas não permitem delimitar se todas são sólidas ou liquidas, podendo, inclusive, ser ambas. Dentre os nódulos existentes, o mais comum que aparece entre as mulheres é o cisto. Os cistos são esferas, que variam de tamanho, cheias de líquido em seu interior.

$\mathrm{Na}$ figura $2 \mathrm{a}$, há uma representação de um cisto pequeno, medindo cerca de $6 \mathrm{~mm}$. Nesse caso, a paciente realizou a mamografia (Figura 2c) e não foi vista nenhuma alteração na imagem. No mesmo dia, realizou a ecografia e foi visto uma região hipoecóica, com suspeita de alteração cística. Foi recomendada que essa paciente fizesse uma imagem por RM de mamas para uma melhor investigação. $\mathrm{Na}$ imagem de RM de mamas (Figura $2 b$ ), na sequência ponderada em T2, houve um realce circular (seta branca) com tamanho significativo, confirmando o cisto.

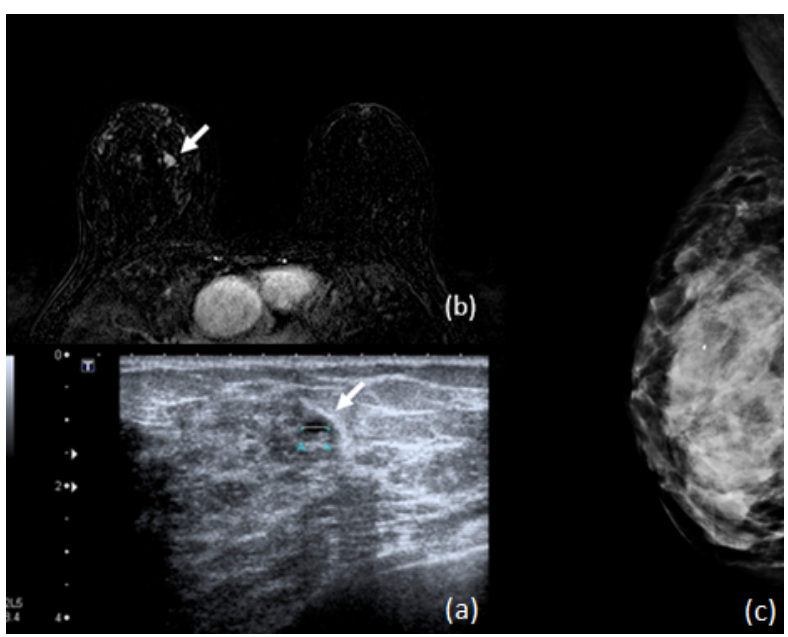

Figura 2. Imagens representativas de um cisto mamário pelas setas na ecografia (a) e na RM (b). Na mamografia (c), tal conteúdo cístico não foi visto. (Fonte: AUTOR, 2017)

A classificação da lesão em maligna, benigna e sem alteração para as 30 pacientes foi quantificada em cada método de diagnóstico por imagem, conforme a Figura 3. Nota-se que nos exames de mamografia e ecografia, não foi possível separar os achados encontrados em cada método, pois, em todos os casos, as pacientes realizaram os exames no mesmo dia e foi feito um único laudo para a mamografia e ecografia.

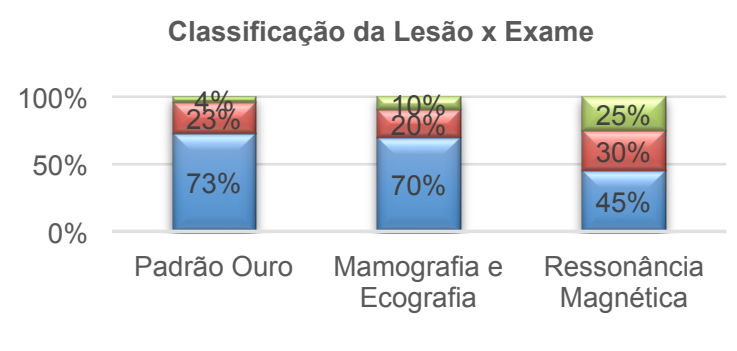

Figura 3. Distribuição do tipo de lesão encontrada no exame de RM, mamografia e ecografia comparado com o padrão ouro (biópsia).

Com a porcentagem de lesão e sem alteração, foi possível determinar os valores de VP, VN, FP e FN para cada exame. A partir das equações 1, 2, 3 e 4, conseguiu-se calcular os valores de sensibilidade, especificidade, valor preditivo positivo (VPP) e valor preditivo negativo (VPN) para mamografia $e$ ecografia e para RM (Tabela 3). Na Tabela 3, LB refere-se às lesões benignas e LM às lesões malignas. 
Tabela 3. Parâmetros de desempenho para os exames de RM, Mamografia e Ecografia.

\begin{tabular}{|c|c|c|c|c|}
\hline & \multicolumn{2}{|c|}{$\begin{array}{l}\text { Mamografia } \\
\text { e Ecografia }\end{array}$} & \multicolumn{2}{|c|}{ RM } \\
\hline & LB & LM & LB & LM \\
\hline Sensibilidade & 0,91 & 0,57 & 0,77 & - * \\
\hline Especificidade & 0,63 & 1 & 1 & - * \\
\hline VPP & 0,87 & 1 & 1 & - * \\
\hline VPN & 0,71 & 0,88 & 0,62 & - * \\
\hline
\end{tabular}

Em relação às indicações para a realização da $\mathrm{RM}$ de mamas, foram analisados todos os 243 requerimentos de janeiro a dezembro do ano de 2016. As justificativas, em sua maioria, não possuíam muitas informações técnicas. Em vários casos, era indicada apenas a necessidade da realização do exame. Por isso, foi importante observar a anamnese dos pacientes e seu histórico de exames. Foi possível organizar as diversas justificativas em 9 categorias: acompanhamento de CA (câncer); acompanhamento após a retirada de um CA; acompanhamento de uma lesão já confirmada como benigna; investigação de um achado em mamografia ou ecografia e com a necessidade de uma verificação mais detalhada; BIRADS 0 em mamografia ou ecografia; paciente com histórico familiar de mama (HF CA); avaliação de prótese; mamas densas ou por mais de um destes motivos descritos (misto). O motivo mais requerido foi investigação mais detalhada (30\%), seguido da avaliação de prótese $(18 \%)$ e histórico familiar de câncer de mama (16\%). O acompanhamento de lesões benignas e mamas densas é o motivo alegado em apenas $1 \%$ dos requerimentos.

\section{Discussão}

Os resultados obtidos nos parâmetros de desempenho (Tabela 4) de sensibilidade, especificidade, valores preditivos positivos $e$ negativos para lesões benignas e malignas dos exames de RM de mamas e a mamografia e ecografia estão, de maneira geral, próximos.

A maior desigualdade obtida entre os exames está na especificidade das lesões benignas (diferença de $37 \%$ ), sendo de $100 \%$ para as imagens por RM e de $63 \%$ para a mamografia e ecografia. A especificidade está relacionada com a capacidade do método indicar a pacientes que não tenham lesão de mama obter realmente seu teste negativo. Esse parâmetro é muito importante, porque se a paciente não tem a lesão e seu resultado é positivo, ela poderá receber determinado tratamento sem a necessidade. $O$ desempenho de $100 \%$ das imagens por RM se tornou um bom indicativo para realização do método em suspeitas de nódulos benignos, uma vez que a especificidade do método é essencial para não submeter as pacientes a tratamentos desnecessários.
Em contrapartida, se a sensibilidade de ambos os métodos é comparada, a RM obteve menor valor $(77 \%)$ que a mamografia e ecografia $(91 \%)$. Na maioria dos casos avaliados em que a RM não detectou a alteração benigna da paciente, a justificativa foi que o nódulo possuía um tamanho de voxel menor que o tamanho da lesão. Ou seja, era menor do que o permitido a ser visto na imagem, indicando a maior sensibilidade da mamografia e da ecografia para pequenas lesões.

A comparação dos parâmetros de desempenho para as lesões malignas não pode ser feita, pois os parâmetros de desempenho da RM não foram significativos para o estudo. Independente do parâmetro, todos seus valores foram de $100 \%$. O motivo desses altos valores não está relacionado com o alto desempenho da técnica, e sim do uso das imagens por RM como complemento à mamografia. O método de rastreio para câncer de mama no Brasil e no mundo é a mamografia. Portanto, se a paciente possui algum tipo de nódulo palpável clinicamente, o primeiro exame que seu médico indicará é a mamografia. Se a paciente for fazer exame para rastreamento, também será a mamografia. Assim, independente do caso, se existe suspeita de nódulo ou não, a primeira imagem a ser visualizada pelo médico será a mamografia. Se houver algum indício de malignidade no nódulo, será realizado uma biópsia para a confirmação através do anátomo-patológico. Por isso, em todos os casos com suspeita de câncer de mama e que a paciente realizou imagens por RM, já havia obtido classificação BIRADS 6 com o resultado do AP. Ou seja, já havia confirmação prévia do câncer de mama. Isso indicou os $100 \%$ em todos os parâmetros de desempenho para as lesões malignas utilizando o método de RM. Para realizar uma melhor análise sobre o exame de RM de mamas para a avaliação de câncer, seria essencial verificar casos, se existentes, de pacientes que realizaram exames de RM antes ou em conjunto com a mamografia.

Em nenhuma das 30 pacientes que realizaram RM de mamas, mamografia e ecografia havia suspeita de microcalcificação. Deve-se ressaltar que o exame de RM de mamas não foi capaz de detectar, pois as microcalcificações possuem tamanhos da ordem de micrômetros, e a resolução espacial do menor voxel encontrado foi no equipamento Avanto, que é de $0,6 \times 0,6 \times 0,9 \mathrm{~mm}^{3}$. Portanto, é correto afirmar que apenas a mamografia é válida para a visualização de microcalcificações.

Os índices dos requerimentos de exames de RM de mamas foram muito úteis para tal estudo para evidenciar o motivo de que as pacientes vinham realizar o exame, uma vez que o padrão ouro para rastreamento de câncer de mama é a mamografia. Dentre eles, o motivo mais comum, a investigação, está relacionado com pacientes que já realizaram algum exame, tanto clínico quanto por imagem, e é necessário a realização do complemento para avaliar melhor a lesão, identificando sua localização, avaliando a composição do tecido ou sua 
vascularização através do uso de contraste, verificar a cadeia linfática e obter a visualização 3D das mamas, dentre outros. A avaliação de prótese, segundo maior motivo de requerimento, é feita devido à recomendação de verificar sua integridade a cada 10 anos, em casos em que a paciente sinta a prótese deslocada ou alguma suspeita de ruptura. Pacientes com histórico familiar de câncer de mama são consideradas de alto risco, dado que aumenta muito a chance de desenvolvimento do tumor, o que justifica o terceiro maior motivo de requerimento das imagens por RM de mamas. Devido a isso, recomenda-se realizar uma vez ao ano a mamografia e, como complemento, a ecografia e a RM de mamas.

Müller-Schimpfle et al. ${ }^{7}$ avaliaram o benefício no diagnóstico de mamas em pacientes que realizaram só mamografia e ecografia em comparação com as que fizeram mamografia, ecografia e RM de mamas. Para tanto, os exames de mamografia e ecografia eram avaliados separadamente da RM e sem o conhecimento do anátomo-patológico. Os autores concluíram que a RM como complemento é bastante válida como método de rastreamento de câncer de mama devida à sua alta sensibilidade (95\%) e, utilizando apenas o método convencional (mamografia e ecografia), esse valor caiu para $83 \%$. Porém, a RM não é indicada em casos em que a paciente tenha alguma suspeita de lesão, uma vez que a especificidade cai de $92 \%$ para $64 \%$ ao utilizar o método combinado.

Moy et al. ${ }^{8}$ conseguiram realizar um estudo semelhante, demonstrando que o exame de RM de mamas foi importante como uma avaliação adicional de um achado mamográfico inconclusivo. O exame de RM não foi avaliado antes da mamografia, porém, como os achados foram inconclusivos, podese avaliar o desempenho de tal exame sem a confirmação da biópsia. Eles utilizaram apenas casos em que a paciente já havia realizado mamografia e ultrassonografia e não havia nenhuma suspeita antes de fazer a RM. Também utilizaram a sensibilidade, especificidade, valor preditivo positivo e valor preditivo negativo, utilizando como padrão ouro o anátomo-patológico e o comportamento da lesão a longo prazo. A sensibilidade encontrada para RM de mamas foi de $100 \%$ e especificidade de $91 \%$. Eles concluíram a importância do exame de RM de mamas como avaliação adicional à mamografia.

O câncer de mama continua sendo a causa mais frequente de morte por câncer entre as mulheres. A mamografia, por décadas vem desempenhando um ótimo papel como rastreamento desta neoplasia. Mas, o avanço de técnicas de exame de mama permitiu demonstrar outras características da glândula, diferentes das já vistas na imagem bidimensional estática. A ecografia nos permite melhor diferenciar lesões sólidas de cistos e informar o tamanho do nódulo. Já nas imagens por $\mathrm{RM}$, é possível visualizar a mama em seus três planos (coronal, sagital e axial), acompanhar dinamicamente a chegada e saída do contraste no tecido e quantificar através de suas curvas de impregnação em lesões. Ao entender melhor seus desempenhos, ou seja, as capacidades de informações que as técnicas de exame por imagem da mama nos podem fornecer e suas respectivas limitações, o diagnóstico médico se torna mais exato. Quanto mais eficaz for o rastreio do câncer de mama ou da alteração benigna, mais rápido poderá ser iniciado o devido tratamento para a paciente.

\section{Conclusões}

Este estudo permitiu quantificar o desempenho dos exames de imagens por RM, mamografia e ecografia. As informações mais relevantes para tal avaliação estão na sensibilidade e especificidade de cada método. Quando se trata de lesões benignas, o exame com maior sensibilidade, ou seja, com a maior probabilidade da paciente com alguma alteração ter realmente seu teste alterado (positivo), foi o de mamografia e ecografia. O método que possuiu maior especificidade, ou seja, maior probabilidade da paciente sem alteração ter realmente seu teste normal (negativo), foi o de imagens por RM. Portanto, considerando os parâmetros encontrados na amostra de 30 pacientes, conclui-se que as imagens por RM de mamas obteve melhor desempenho para avaliação das lesões benignas do que os exames de mamografia e ecografia. No que diz respeito às lesões malignas, os parâmetros de desempenho encontrados não são válidos para avaliar o desempenho da RM.

Em estudos futuros, pretende-se ampliar esta pesquisa, a fim de entender melhor a eficiência das imagens por RM de mamas para rastreamento de lesões malignas e o desempenho individual da mamografia e da ecografia.

\section{Referências}

1. Ministério da saúde. Instituto Nacional de Câncer José Alencar Gomes da Silva (INCA). Diretrizes para detecção precoce do câncer de mama no Brasil. Rio de Janeiro:2015.

2. International Atomic Energy Agency (IAEA) Human Health Series No. 17: Quality Assurance Programme for Digital Mammography. Vienna:2011.

3. Cardenosa G. Clinical Breast Imaging: The Essentials. Wolters Kluwer Health: First edition; 2014.

4. Kuhl C. The current status of breast MR imaging part I. Choice of technique, image interpretation, diagnostic accuracy, and transfer to clinical practice. Radiology 2007; 244 (2): 356-378.

5. Rausch DR, Hendrick RE. How to optimize clinical breast MR imaging practices and techniques on your 1.5-T system. Radiographics 2006; 26(5): 1469-1484

6. Bellusci SM. Epidemiologia. Editora Senac São Paulo; 1995.

7. Müller-Schimpfle M, Stoll P, Stern W, Kurz, S, Dammann F Claussen CD. Do mammography, sonography, and MR mammography have a diagnostic benefit compared with mammography and sonography?. American journal of roentgenology 200; 168(5): 1323-1329.

8. Moy L, Elias K, Patel V, Lee J, Babb JS, Toth, HK, Mercado $\mathrm{CL}$. Is breast MRI helpful in the evaluation of inconclusive mammographic findings?. American Journal of Roentgenology 2009; 193(4): 986-993.

9. American College of Radiology (ACR) BI-RADS $B$ Committee. ACR BI-RADS Breast Imaging Reporting and Data System: Breast Imaging Atlas, Mammography, Breast Ultrasound, Magnetic Resonance Imaging; 2013.

10. Andolina V, Lillé S. Mammographic Imaging: A Practical Guide. Lippincott Williams \& Wilkins; 2011. 
11. Berg WA, Gutierrez L, NessAiver MS, Carter WB, Bhargavan $M$, Lewis RS, loffe OB. Diagnostic accuracy of mammography, clinical examination, US, and MR imaging in preoperative assessment of breast cancer. Radiology 2004; 233(3): 830-849.

12. Boetes C, Mus RD, Holland R, Barentsz JO, Strijk SP, Wobbes T, Ruys SH. Breast tumors: comparative accuracy of MR imaging relative to mammography and US for demonstrating extent. Radiology 1995; 197(3): 743-747.

13. Bone B, Aspelin P, Bronge L, Isberg B, Perbeck L, Veress B. Sensitivity and specificity of MR mammography with histopathological correlation in 250 breasts. Acta radiologica 1996; 37(2): 208-213.

14. Kilic F, Ogul H, Bayraktutan U, Gumus H, Unal O, Kantarci M, Yilmaz $\mathrm{MH}$. Diagnostic magnetic resonance imaging of the breast. The Eurasian journal of medicine 2012; 44(2): 106.

15. Názaro ACP, Rego MF. Nódulos benignos da mama: uma revisão dos diagnósticos diferenciais e conduta. Revista Brasileira Ginecologia e Obstetrícia 2007; 29(4): 211-9.

\section{Contato:}

Carolina Moreira Maulaz

Av. Ipiranga, 6681 - Partenon, Porto Alegre - RS

E-mail: carolina.maulaz@acad.pucrs.br 